\title{
Metabolic Syndrome in Obese Children over Five Years Old
}

\author{
Douaa Kharbotly ${ }^{1}$, Ahmad Chreitah ${ }^{1}$, Muhammed Imad Khayat ${ }^{2}$ \\ ${ }^{1}$ Department of Pediatrics, Faculty of Medicine, Tishreen University Hospital, Lattakia, Syria \\ ${ }^{2}$ Department of Laboratory Medicine, Tishreen University Hospital, Lattakia, Syria
}

Email address:

douaakh88@gmail.com (D. Kharbotly),Ahmad.Chreitah@tishreen.edu.sy (A. Chreitah),

MohammadImadKhaiat@gmail.com (M. I. Khayat)

\section{To cite this article:}

Douaa Kharbotly, Ahmad Chreitah, Muhammed Imad Khayat. Metabolic Syndrome in Obese Children over Five Years Old. American Journal of Pediatrics. Vol. 7, No. 3, 2021, pp. 113-116. doi: 10.11648/j.ajp.20210703.14

Received: June 9, 2021; Accepted: June 21, 2021; Published: July 9, 2021

\begin{abstract}
Background: Obesity is an important pediatric public health problem associated with increased risk of many complications in childhood and increased morbidity and mortality throughout adult life. Objectives: To assess the prevalence of Metabolic Syndrome in obese children and the relationship between Metabolic Syndrome and Body mass index (BMI), sex, age and family risk factors like obesity, hypertension, diabetes mellitus and cardiovascular disease. Materials and Methods: An analytic cross-sectional study was conducted in 178 overweight and obese children and adolescents aged 5-16 years old seen at the General Pediatric Clinic of the Tishreen University Hospital between March 2020 and February 2021. Weight, high, waist Circumference and blood pressure were measured. Blood samples were taken after 12 hours fasting and fasting blood glucose (FBG), total cholesterol (T Cho), high- density lipoprotein cholesterol (HDL) and low- density lipoprotein cholesterol (LDL) were measured. The Metabolic Syndrome MS was diagnosed based on pediatric and adolescent criteria adapted from the National Cholesterol Education Program Third Adult Treatment Panel (NCEP-ATP III) definition. Results: A total of 178 children, 82 males $(46.1 \%)$ and 96 females $(53.9 \%)$ with mean age $9.9 \pm 2.5$ SD were in our study. Out of the 178 children, $62(34.8 \%)$ were overweight, 101 (56.7\%) obese and 15 (8.4\%) were severe obese. According to the NCEP ATP III, (32.6\%) out of (178) children were diagnosed with MS. Among children with MS, most components of MS were increased waist circumference WC (93.1\%), increased TG (86.2\%), reduced HDL (51.7\%), increased BP (50\%) and increased FBS (25.9\%). MS prevalence was $12.9 \%, 36.6 \%, 86.7 \%$ in overweight, obese and severe obese children respectively
\end{abstract}

Keywords: Metabolic Syndrome, Obese, Overweight, Cardiovascular Disease

\section{Introduction}

The World Health Organization WHO has considered overweight and obesity a global epidemic among children since 1998 [1]. Once considered a high -income country problem, overweight and obesity are now on the rise in lowand middle-income countries [2]. Childhood obesity has immediate comorbidities including type 2 diabetes, hypertension, hyperlipidemia and alcoholic fatty liver disease. Obesity is the result of a complex effects among genetics factors, caloric intake, physical activity and energy consumption. Insulin resistance increases with increasing obesity and has effects of lipid metabolism and cardiovascular health [3]. Metabolic Syndrome MS is a group of metabolic risk factors for cardiovascular disease and type 2 diabetes. These risk factors occurs especially in overweight and obese [4].

There is a relation between obesity, insulin resistance IR and many abnormalities such as type 2 diabetes, hypertension and dyslipidemia, all these mechanisms are responsible for MS [5]. There are many definitions for MS and it is a subject of discussion among children and adolescents. The most used definition is National Cholesterol Education Program Adult Treatment Panel Third (NCEP-ATP III) definition. No studies had been conducted in Syria to establish the prevalence of Metabolic Syndrome among children over five years old.

\section{Materials and Methods}

\subsection{Study Population}

After approval by the local research ethics committee, an analytic cross-sectional study was conducted in 178 
overweight and obese children and adolescents aged 5-16 years old seen at the General Pediatric Clinic of Tishreen University Hospital between March 2020 and February 2021.

Inclusion Criteria were as follows: children over five years old with body mass index $\mathrm{BMI} \geq 85$ percentile.

Non-Inclusion Criteria

Hypothyroidism, cushing syndrome, type I diabetes, being on medications or having a condition known to influence insulin action or insulin secretion (e.g. glucocorticoid therapy) as indicated by parents.

Children who met inclusion criteria were classified according to their BMI percentile ( $\mathrm{p}$ ) according to $\mathrm{CDC}$ as follows:

Overweight with BMI $\geq 85 \mathrm{p}$ and $<95 \mathrm{p}$, obese with BMI $\geq$ $95 \mathrm{p}$ and $<99 \mathrm{p}$ and severe obese with $\mathrm{BMI} \geq 99 \mathrm{p}$. Weight was measured with the child in the center of a scale in light clothing and without shoes. Height was measured with the child standing with back leaning against the stadiometer of the same scale. Waist Circumference (WC) was measured at the level of the umbilicus with the child standing and breathing normally. It is defined as $\mathrm{WC} \geq 90$ percentile for age and sex using WC percentiles for European -American children and adolescents. Blood pressure was measured with the auscultatory method using appropriate cuff after 20 minutes of rest. It was measured three times and the mean of the last two values was taken. It was considered high if systolic or diastolic pressure was $\geq 90$ percentile using American Academy of Pediatrics AAP tables in 2017 according to the age, sex and height [6]. Blood samples were taken after 12 hours fasting and fasting blood glucose (FBG), total cholesterol (T Cho), high- density lipoprotein cholesterol (HDL) and low- density lipoprotein cholesterol (LDL) were measured.

The Metabolic Syndrome MS was diagnosed based on pediatric and adolescent criteria adapted from the National Cholesterol Education Program Adult Treatment Panel Third (NCEP-ATP III) definition.

Children meeting three or more of the following criteria were diagnosed with $\mathrm{MS}: \mathrm{FBG} \geq 100 \mathrm{mg} / \mathrm{dl}, \mathrm{TG} \geq 110 \mathrm{mg} / \mathrm{dl}$,
$\mathrm{WC} \geq 90$ th percentile for age and sex, systolic or diastolic blood pressure $\geq 90$ th percentile for age and sex and HDL cholesterol $\leq 40 \mathrm{mg} / \mathrm{dl}$ [7].

\subsection{Statistical Analysis}

The continuous variables were presented as (mean, standard deviation) and categorical variables as (frequency, percentage).

Prevalence Rate was calculated and chi-square test was used to study the relation between categorical variables. To compare between categorical variables, we used Pearson's Chi-square test. A p-value $\leq 5 \%$ was considered statistically significant. All statistical analyses were conducted with the use of the Statistical Package for Social Sciences (SPSS) Version 20.

\section{Results}

A total of 178 children, 82 males (46.1\%) and 96 females (53.9\%) with mean age $9.9 \pm 2.5 \mathrm{SD}$ were in our study. Out of the 178 children, $62(34.8 \%)$ were overweight, $101(56.7 \%)$ obese and $15(8.4 \%)$ were severe obese. According to the NCEP ATP III, 58 of the 178 children (32.6\%) were diagnosed with MS. One out of every three obese children have Metabolic Syndrome. Out of 82 boys 29 (35.36\%) was diagnosed with MS, and out of 96 girls 29 (30.2\%) was diagnosed with MS. There was no difference between MS prevalence between boys and girls ( $\mathrm{P}$ value $=0.4$ ). Among children with MS, most components of MS were increased waist circumference WC (93.1\%), increased TG (86.2\%), reduced HDL $(51.7 \%)$, increased BP $(50 \%)$ and increased FBS (25.9\%) (table 1). The most prevalent diagnostic criteria of MS was increased WC (93.1\%) followed by hyper TG (86.2\%). MS prevalence was $12.9 \%, 36.6 \%, 86.7 \%$ in overweight, obese and severe obese respectively. MS prevalence increased with higher BMI values (P value $=0.0001)$ (table 2$)$.

Table 1. Number and Percentage of children with MS meeting the different diagnostic criteria.

\begin{tabular}{lll}
\hline Dx Criteria & With Metabolic Syndrome & Without Metabolic Syndrome \\
\hline 1WC & $54(93.1 \%)$ & $39(32.5 \%)$ \\
1TG & $50(86.2 \%)$ & $50(41.7 \%)$ \\
च $\mathrm{HDL}$ & $30(51.7 \%)$ & $9(7.5 \%)$ \\
« $\mathrm{BP}$ & $29(50 \%)$ & $13(10.8 \%)$ \\
1 $\mathrm{GLU}$ & $15(25.9 \%)$ & $9(7.5 \%)$ \\
\hline
\end{tabular}

Dx criteria: Differential diagnosis.

Table 2. Prevalence of MS according to degree of obesity.

\begin{tabular}{lll}
\hline BMI & With MS & Without MS \\
Overweight & $8(12.9 \%)$ & $54(87.1 \%)$ \\
Obese & $37(36.6 \%)$ & $64(63.4 \%)$ \\
Severe Obese & $13(86.7 \%)$ & $2(13.3 \%)$ \\
\hline
\end{tabular}

BMI: Body Mass Index, MS: Metabolic Syndrome.

We studied the relationship between WC and (TG, HDL, BP and GLU) with Pearson correlation coefficient. We found positive linear relationship between $\mathrm{WC}$ and TG, BP and GLU and negative linear relationship between WC and HDL (table 3).
There was a large positive correlation between $\mathrm{WC}$ and $\mathrm{TG}$, medium positive correlation between $\mathrm{WC}$ and $\mathrm{BP}$ and small positive correlation between WC and GLU. There was a medium negative correlation between $\mathrm{WC}$ and $\mathrm{HDL}$. That 
means when WC increased, TG, BP and FBS valued were increased and when WC increased, HDL values were reduced.

Table 3. The relationship between $W C$ and (TG, BP, GLU and HDL).

\begin{tabular}{lll}
\hline & Pearson Correlation & P-value \\
\hline TG & 0.6 & 0.003 \\
BP & 0.5 & 0.0001 \\
HDL & -0.4 & 0.005 \\
GLU & 0.3 & 0.01 \\
\hline
\end{tabular}

Based on age, there was no difference in the prevalence of MS between the age groups $(\mathrm{P}$ value $=0.5)$.

MS prevalence was higher in children with positive family risk factors like obesity, hypertension, diabetes mellitus and cardiovascular disease (table 4).

Table 4. The relationship between MS prevalence and family risk factors.

\begin{tabular}{lll}
\hline Family risk factors & Without MS & With MS \\
\hline Positive & $19(15.8 \%)$ & $50(86.2 \%)$ \\
Negative & $101(84.2 \%)$ & $8(13.8 \%)$ \\
\hline
\end{tabular}

MS: Metabolic Syndrome.

\section{Discussion}

The pathophysiology of MS begins early in children and cardiovascular disease CVD as a consequence appears in small adults. Early diagnosis of MS and identifying risk factors in children (silent phase) and early intervention may help controlling MS and its complications.

In our study, MS prevalence was $32.6 \%$ in 178 obese children between 5-16 years old. Almost one third of obese children have MS. The most common component of MS was increased WC and the least common is increased FBS. In this study half of the children with MS have reduced HDL which is considered the most important risk factor for increased cardiovascular risk in children with MS. Quite the opposite higher HDL levels are considered a preventive factor of MS in obese children [8]. The least common component of MS was increased FBS. Maybe that is because Glucose $\geq 100$ $\mathrm{mg} / \mathrm{dl}$ is rare in children [9]. MS prevalence increased directly with increased degree of obesity. MS prevalence was significantly higher in children with positive family risk factors like obesity, hypertension, diabetes mellitus and cardiovascular disease. This finding reflects the effect of genetic factors, similar lifestyle and nutritional habits which influenced by socioeconomic, cultural factors and individual choices [10].

Basma et al reported that the MS prevalence was $23.2 \%$ in 146 obese children between 6-18 years old. Reduced HDL was the most common component of MS [11]. According to Syed Mahboob in United Arab Emirates, 8.9\% of 284 normal, overweight and obese children were diagnosed with MS based on NCEP definition. MS prevalence was $4.5 \%$ in normal weight children, $16.7 \%$ in overweight children and $30 \%$ in obese children [12]. That was similar to our finding that MS prevalence increased with increased degree of obesity. In Mexico, Oralia et al found that the prevalence of MS was $22.6 \%$ and increased WC was the most common component of MS which is similar to our finding [13]. In our study, increased FBG was the least common, this finding was similar in all the previous studies cited above. That indicates that FBG $\geq 100$ $\mathrm{mg} / \mathrm{dl}$ as a criteria for MS is not appropriate for detecting abnormalities in glucose metabolism in overweight and obese children [14].

\section{Conclusion}

Our study showed a high prevalence of Metabolic Syndrome in obese children and increased prevalence with increased BMI values. Central obesity and dyslipidemia were the most important risk factors for MS among children. MS prevalence was significantly higher in children with positive family risk factors like obesity, hypertension, diabetes mellitus and cardiovascular disease. Strong measures should be done to reduce obesity prevalence in children. Early diagnosis of MS and identifying risk factors in children (silent phase) and early intervention may help controlling MS and its complications.

\section{Recommendations}

We emphasize the importance of investigating the components of Metabolic Syndrome within the initial investigations for every obese child.

Follow_up of negative MS children and revealing the percentage of their conversion to positive MS. Determining the response of positive MS children to treatment and detecting new metabolic complications،if any.

\section{Declarations}

\section{Competing of Interests}

All the authors do not have any possible conflicts of interest.

\section{Ethical Considerations}

All the children freely accepted to be part of the study and the permission from parents was taken.

\section{Availability of Data and Materials}

Most of the data were in this manuscript. Other data can be asked from corresponding author.

\section{Funding}

Not applicable.

\section{Author Contributions}

All authors performed the measurements and wrote the manuscript. Literature review was done by Dr. Douaa Kharbotly, and both authors performed the analytic calculations and performed the numerical simulations. 


\section{Acknowledgements}

We wish to thank all doctors in the pediatric department. We want to thank Miss Elissar Hejazia for technical help.

\section{References}

[1] Vaquero Alvarez, M., Aparicio-Martinez, P., Fonseca Pozo, F. J., Valle Alonso, J., Blancas Sánchez, I. M., \& RomeroSaldaña, M. (2020). A sustainable approach to the metabolic syndrome in children and its economic burden. International journal of environmental research and public health, 17 (6), 1891.

[2] Lee, K. (2008). The World Health Organization (WHO). Routledge.

[3] St Geme, J. W., Blum, N. J., Shah, S. S., Tasker, R. C., \& Wilson, K. M. (2020). Nelson textbook of pediatrics.

[4] Klish, W. J., Kirkland, J., \& Motil, K. (2015). Comorbidities and complications of obesity in children and adolescents. Up To Date [Internet].(Consultado noviembre 2009). Disponible en: http://www.uptodate.com/contents/comorbidities-andcomplications-of-obesity-in-children-and-adolescents.

[5] Mantzoros, C. (2005). Insulin resistance: Definition and clinical spectrum. Up to Date Online, 14, 1-5.

[6] Mattoo, T. K. (2009). Definition and diagnosis of hypertension in children and adolescents. Up To Date.

[7] DeBoer, M. D. (2019). Assessing and managing the metabolic syndrome in children and adolescents. Nutrients, 11 (8), 1788.
[8] Özer, S., Kazanc, N. Ö., Sönmezgöz, E., Karaaslan, E., Altunta, B., \& Kuyucu, Y. E. (2015). Higher HDL levels are a preventive factor for metabolic syndrome in obese Turkish children. Nutricion hospitalaria, 31 (1), 307-312.

[9] Weiss, R., Dziura, J., Burgert, T. S., Tamborlane, W. V., Taksali, S. E., Yeckel, C. W.,... \& Caprio, S. (2004). Obesity and the metabolic syndrome in children and adolescents. New England journal of medicine, 350 (23), 2362-2374.

[10] Sangun, Ö., Dündar, B., Köşker, M., Pirgon, Ö., \& Dündar, N. (2011). Prevalence of metabolic syndrome in obese children and adolescents using three different criteria and evaluation of risk factors. Journal of clinical research in pediatric endocrinology, 3 (2), 70.

[11] Damiri, B., Alhala, A. A., Najjar, L., \& Alqadome, S. (2018). Metabolic syndrome and its risk factors among overweight and obese Palestinian schoolchildren using IDF and NCEPATP/III definitions. Ann Clin Lab Res, 6 (3), 242.

[12] Shah, S. M., Aziz, F., Al Meskari, F., Al Kaabi, J., Khan, U. I., \& Jaacks, L. M. (2020). Metabolic syndrome among children aged 6 to 11 years, Al Ain, United Arab Emirates: Role of obesity. Pediatric diabetes, 21 (5), 735-742.

[13] Medinaa, O. N., Arriagaa, R. V., Valverdeb, L. F., Lópeza, C. P. R., Skidmorec, O. M., Torresd, M. C. G.,... \& Lópeza, S. G. (2015). Prevalence of metabolic syndrome in overweight and obese schoolchildren.

[14] Rodrigues, L. G., Mattos, A. P., \& Koifman, S. (2011). Prevalence of metabolic syndrome in overweight and obese outpatient children and adolescents: comparative analysis using different clinical definitions. Revista Paulista de Pediatria, 29, 178-185. 